\title{
Structural femtochemistry: Experimental methodology
}

\author{
J. C. Williamson and A. H. Zewail \\ Arthur Amos Noyes Laboratory of Chemical Physics, California Institute of Technology, Pasadena, CA 91125 \\ Contributed by A. H. Zewail, March 12, 1991
}

\begin{abstract}
The experimental methodology for structural femtochemistry of reactions is considered. With the extension of femtosecond transition-state spectroscopy to the difiraction regime, it is possible to obtain in a general way the trajectories of chemical reactions (change of internuclear separations with time) on the femtosecond time scale. This method, considered here for simple alkali halide dissociation, promises many applications to more complex reactions and to conformational changes. Alignment on the time scale of the experiments is also discussed.
\end{abstract}

Over the last 6 years, progress has been made in probing the femtosecond chemistry (femtochemistry) of isolated reactions in real time (for reviews, see refs. 1 and 2). The temporal dynamics of the nuclear motion are resolved on the femtosecond time scale and, hence, bond breaking and bond forming processes can be observed as reagents change to products in the transition state and transition-state region (3). Studies of elementary reactions in gas phase and molecular beams have so far included the following dynamical processes (2): bond dissociation, resonance oscillation, bound motion, and saddle-point transition states. With picosecond resolution (and soon femtosecond), the formation of a product in a bimolecular reaction was also studied in real time (1, 2). Recently, the femtosecond dynamics of autoionization (4) and of highly excited molecules (5) have been examined by using these techniques.

The success of these experiments relies on the use of femtosecond optical pump and probe pulses combined with very sensitive gas phase and molecular beam detection techniques, laser-induced fluorescence, and multiphoton ionization/mass spectrometry. These femtosecond transitionstate spectroscopies (FTS) $(1,2)$ enable one to identify the fragment molecules both in the course of the reaction (transition states) and as they become free (nascent products). Absorption kinetics of the fragments on the subpicosecond time scale has also been introduced to study dissociation in the gas phase (ref. 6 and references therein).

In all of the FTS experiments, the key advance lies in the ability to probe the transition region of chemical reactions by monitoring changes in the potential energy with time. Much theoretical work by many research groups (see references in the reviews of refs. 1 and 2) has been done to compare with experiments and to learn about details of the dynamics and the potential energy surface. In a simple reaction involving two (and three) atoms, theory has been tested for experiments probing the temporal dynamics of the motion, the nature of the wave packet at different times, and the potential energy as a function of internuclear separation. Recently, Mokhtari et al. (7) have reported on an experimental method for obtaining the trajectories of the changes of the internuclear distance of the reaction coordinate [one, $R(t)$, in this case] with time. For more complex reactions, a general

The publication costs of this article were defrayed in part by page charge payment. This article must therefore be hereby marked "advertisement" in accordance with 18 U.S.C. $\$ 1734$ solely to indicate this fact. method is needed for obtaining $R(t)$ on the multidimensional potential energy surface, which involves all atoms in motion.

A proposal was made recently to "replace" the optical probe pulse in FTS by an electron pulse (8). The resulting diffraction pattern would reveal the changes in $R$ for all atoms with femtosecond time resolution. This femtosecond transition-state diffraction (FTD) (8) will enable us to obtain structural femtochemistry on a variety of reactions and will complement FTS in its molecular state selection. To one of the experimental setups at California Institute of Technology, which already houses the femtosecond/molecular beam apparatuses, we are adding an electron source and a diffraction compartment. The "machine" will thus be equipped for studies of the spectroscopy (laser-induced fluorescence and time-of-flight MS) and the structural dynamics (electron diffraction) of reactions in gas phase and molecular beams (see Fig. 1). In this paper, we describe the details of such apparatus, the sensitivity, and our first examples for study.

\section{FTS and FTD}

Discussion of FTS and its applications can be found in refs. 1,2 , and 8. Basically, in these experiments, the reaction is initiated at $t=0$ by a pump pulse $\left(\lambda_{1}\right)$, thus establishing a known total energy in the bond. A second, delayed probe pulse of different wavelength $\left(\lambda_{2}\right)$ monitors the dynamics of the nuclear motion (e.g., dissociation) as the resulting fragments separate from each other. For a diatomic molecule (e.g., A-B), the free fragments A and B have different spectra (or ionization potentials) from that of the activated complex $[A \cdot B]^{\ddagger *}$ of the transition region.

The experiments on the $\mathrm{NaI}$ dissociation reaction $(7,9)$ illustrate these concepts (8) (see Fig. 2). When $[\mathrm{Na} \cdot \mathrm{I}]]^{\ddagger *}$ is monitored, one observes the formation and decay of the complex. The bond is partly covalent and partly ionic, and the oscillation of the complex between the two characters can be observed. These experiments at different $\lambda_{1}$ and $\lambda_{2}$ map out the change of the internuclear distance $R$ with time $t$. If, instead, free $\mathrm{Na}$ atoms are detected, at a different $\lambda_{2}$ and different time delays, then one observes "steps" of $\mathrm{Na}$ atom buildup.

The same type of experiments can now be performed by using an electron probe pulse whose wavelength is determined by the de Broglie relationship: $\lambda=h /(2 m e V)^{1 / 2}$, where $h$ is Planck's constant and $m$ and $e$ are the electron mass and charge, respectively (without relativistic effects). Including relativistic effects, which are small, $\lambda \approx 0.05 \AA$ for an accelerating voltage of $V \approx 50 \mathrm{kV}$. In this regime of highenergy electron diffraction, the $R$ resolution is very good (see below). The temporal resolution is determined by the optical probe pulse and other factors related to electron dispersion and focusing, as discussed below. The $\lambda_{1}, \lambda_{2}$ pulses used in our current FTS apparatus will still be part of the experiment: the $\lambda_{1}$ pulse initiates the reaction and $\lambda_{2}$ (optical) creates the $\lambda_{2}$ (electron). Thus, the delay time between the $\lambda_{1}$ pulse and

Abbreviations: FTS, femtosecond transition-state spectroscopy(ies); FTD, femtosecond transition-state diffraction; ERD, experimental radial distribution. 


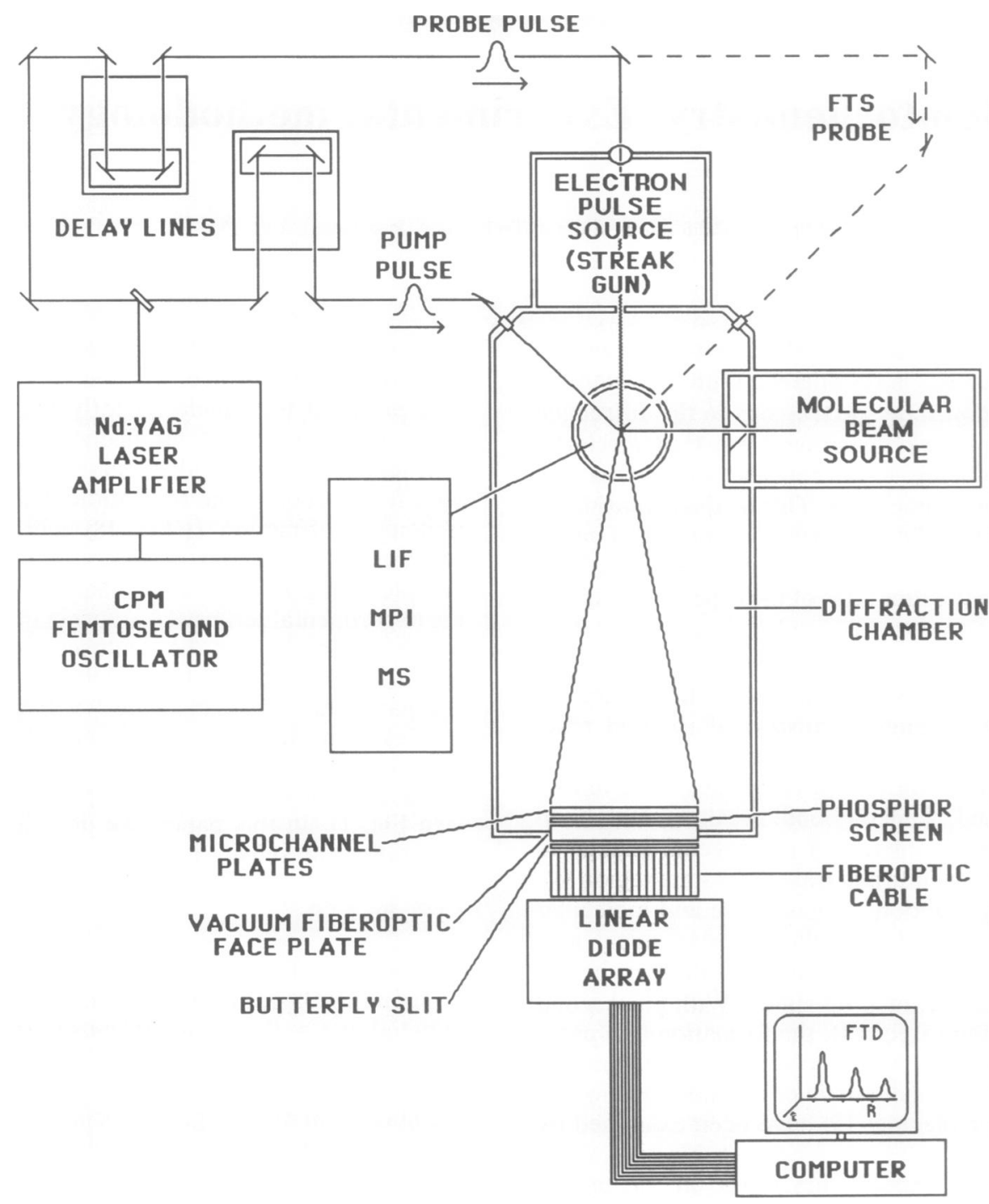

FIG. 1. A representation of the FTD apparatus. The pump pulse establishes the $t=0$ of the reaction in the molecular beam. A femtosecond electron burst, generated by the probe pulse, is scattered by the molecular beam, and the resulting diffraction pattern is detected by using a linear diode array. FTS experiments may be conducted by sending the probe pulse directly into the chamber and using laser-induced fluorescence or time-of-flight MS. CPM, colliding-pulse mode-locked; LIF, laser-induced fluorescence; MPI, multiphoton ionization.

$\lambda_{2}$ (electron) can be controlled and varied to record snapshots of the actual nuclear motion. For $\mathrm{NaI}$ as an example, complete separation of $\mathrm{Na}$ and I takes $\approx 10 \mathrm{ps}$ (about 10 resonance oscillations between the covalent and ionic potentials), and this time will be observable directly in the diffraction pattern as discussed below (in Applications). Extension to other systems should be straightforward.

\section{Experimental Apparatus}

Femtosecond Optical Pulse/Molecular Beam System. The experimental setup for the laser systems has been described in detail before (1-3). A femtosecond oscillator (collidingpulse mode-locked) was built at California Institute of Technology following the pioneering design of Shank and colleagues (ref. 10 and references therein). A Nd:YAG-pumped four-stage dye amplifier seeded by this colliding-pulse modelocked dye laser is used to obtain $\approx 50$-fs pulses with energies of $\approx 0.5 \mathrm{~mJ}$. Part of the beam is used to generate a continuum, and wavelengths are selected by using interference filters. Amplification of the filtered light, as well as nonlinear mixing and doubling techniques, is used to finally generate the $\lambda_{1}$ and $\lambda_{2}$ pulses of interest (1-3).
The probe pulse is delayed in time, with a Michelson interferometer, relative to the pump pulse. Both pump and probe beams are recombined and focused onto the molecular beam; the relative polarization is well-defined. The molecular beam is equipped with a time-of-flight MS and a laser-induced fluorescence compartment. The modifications for FTD are discussed below.

The Femtosecond Electron Pulse. The generation and recording of ultrashort electron and $x$-ray bunches has many applications. For example, a picosecond x-ray framing camera has been developed for imaging the soft $x$-rays that are emitted from laser-produced plasmas (ref. 11; ref 12 and references therein). The generation of picosecond $x$-ray pulses is now under intensive investigation (see refs. 13 and 14 and the articles in ref. 15).

Mourou, Williamson, and El-Sayed-Ali $(16,17)$, in a pioneering effort, have shown that a modified streak camera can be used to generate a picosecond electron diffraction pattern from a film or surface (16-18). Streak cameras are conventionally used to image the intensity distribution of ultrashort light pulses (19-21). A light pulse enters the streak camera, falls on a photocathode, and produces an electron burst. The burst is immediately accelerated across a potential difference of several kilovolts and is focused through a deflection 


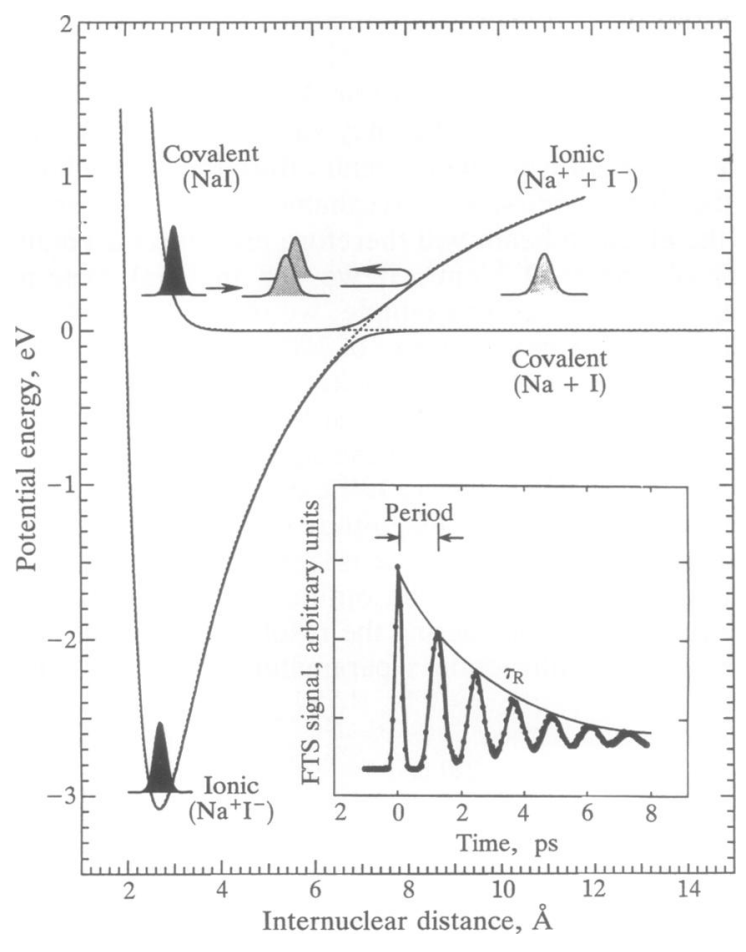

FIG. 2. A schematic of the wave packet motion in the dissociation reaction of $\mathrm{NaI}$. The femtosecond pump pulse starts the reaction ( $t$ $=0$ ) by preparing the molecules on the adiabatic potential. This potential is formed by the avoided crossing of the ionic and covalent curves. The wave packet then oscillates back and forth, decaying $(\approx 10 \%)$ to $\mathrm{Na}+\mathrm{I}$ every time it approaches the crossing region. In FTS, the femtosecond probe pulse monitors this oscillatory motion and the decay $\left(\tau_{R}\right)$, as shown in the Inset (see ref. 9).

electrode. A sweep voltage, carefully synchronized with the arrival of the electron burst, is applied across the deflection electrode. This spreads the electrons perpendicularly to their direction of travel and converts the temporal distribution of the electrons into a spatial distribution. After passing through a cone electrode (used to eliminate spurious signals), the electrons collide with a phosphor plate and produce an image of the light pulse.

By removing the deflection and cone electrodes and adding a thin aluminum scatterer, it was possible to generate a transmission diffraction pattern from a 100 -ps electron burst (16). Investigations have continued with reflection highenergy electron diffraction on the picosecond time scale (18).

Recent improvements in streak camera technology indicate that we should be able to generate electron diffraction patterns with femtosecond resolution. A 500-fs streak camera was described by Kinoshita et al. (22) and is now commercially available from Hamamatsu, Middlesex, NJ. A recent advance of a 50 -fs streak camera $(23,24)$ has also been reported. These new streak cameras will serve as electron beam sources for our probe pulse in the femtosecond electron diffraction experiments; they will also be part of the FTS apparatus.

Difiraction Pattern Detection. Once a femtosecond burst of electrons has been generated and scattered through the molecular beam at a given delay time, the diffraction pattern must be detected. Traditionally, a photographic plate is placed adjacent to the phosphor screen and exposed to the diffraction pattern for seconds or even minutes (25). After the photographic plate is developed, the fine details of the diffraction pattern are obtained by using a microdensitometer. A rotating sector is often placed between the scattering sample and the phosphor screen to compensate for the radial drop-off in intensity.
The exposure time may be dramatically reduced with amplification. Electron diffraction patterns are amplified with either image intensifiers, which amplify the light produced by the phosphor screen, or microchannel plates, which multiply the number of electrons incident on the phosphor screen. A microchannel plate typically provides a gain of $10^{4}$, and Sainov (26) reports that if two microchannel plates are placed in a chevron configuration, a gain of $10^{8}$ is possible. Mourou and Williamson (16) were able to photographically record the diffraction pattern of a film from a single 100 -ps burst of electrons by using an image intensifier with a gain of $10^{4}$.

An alternative to recording diffraction patterns on photographic plates is also known (27). A fiber-optic vacuum faceplate transfers a diameter slice of the diffraction pattern from the phosphor screen to a photodiode array via a fiber-optic cable. The photodiode array interfaces directly with a computer; it allows immediate observation of a diffraction pattern. In addition, a butterfly slit placed between the faceplate and the cable duplicates the effects of a rotating sector (28). This detection apparatus allows for high repetition rates and for reproducibility between experiments, and this system is ideal for FTD (see Fig. 1).

\section{Performance Specifications}

Electron energy, temporal resolution, and sensitivity are the three factors that are important for FTD experiments. Here, we consider these factors.

Electrons with energies of the order of $40 \mathrm{keV}$ to $100 \mathrm{keV}$ are typically used in diffraction experiments. A 20-keV source of electrons (16) was used to create a 100-ps diffraction pattern of aluminum foil, and it is not unreasonable to expect that the 10-keV electrons produced in the Kinoshita tube (22) will contain enough energy for FTD. Higher energy electrons might be produced with this tube by increasing the accelerating voltage, but it may be necessary to move the photocathode further from the electrode in order to maintain

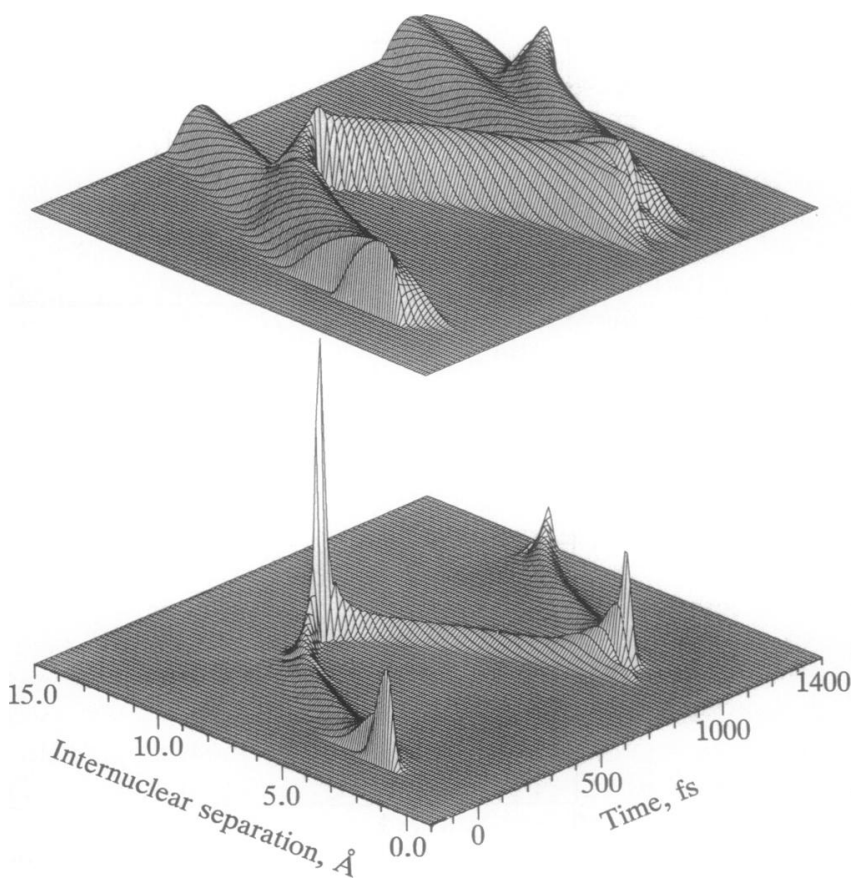

FIG. 3. Simulated ERD curve for excited $\mathrm{NaI}$ as a function of time. Classical mechanical calculations were made by using potential curves described in ref. 9. (Lower) Relative intensities. (Upper) Logarithm of the relative intensities. Dissociation of $\mathrm{Na}+\mathrm{I}$ along the covalent curve is clearly visible in Upper. 
the $6 \mathrm{kV} / \mathrm{mm}$ level described by Kinoshita. In our apparatus, we will use voltages in this range and determine the optimum time resolution (50-500 fs).

The electron beam used in FTD must have a well-defined energy; an upper uncertainty limit of $0.1 \%$ has been suggested for diffraction in general. For a pulse of 50- to 500-fs duration, the energy uncertainty indicates that the electrons are of the same energy to within one part in $10^{4}-10^{6}$.

A femtosecond pulse with energy of sub-millijoules will be used in an FTD experiment. This energy is higher than the energy used in picosecond electron pulse generation (16). For the picosecond pulse generation, using 266-nm light and a photocathode with a quantum efficiency of the order of $10^{-5}$, $\approx 10^{8}$ electrons are produced. The number of electrons that undergo single elastic collisions in a sample may be determined by comparing $\mu_{\mathrm{s}}$, the mass thickness parameter, with the product of the sample's thickness and density (29). For $20-\mathrm{keV}$ electrons incident on aluminum, $\mu_{\mathrm{s}}$ is $3 \times 10^{-3}$ $\mathrm{mg} / \mathrm{cm}^{2}$, and a $150-\AA$-thick aluminum foil is sufficiently thin
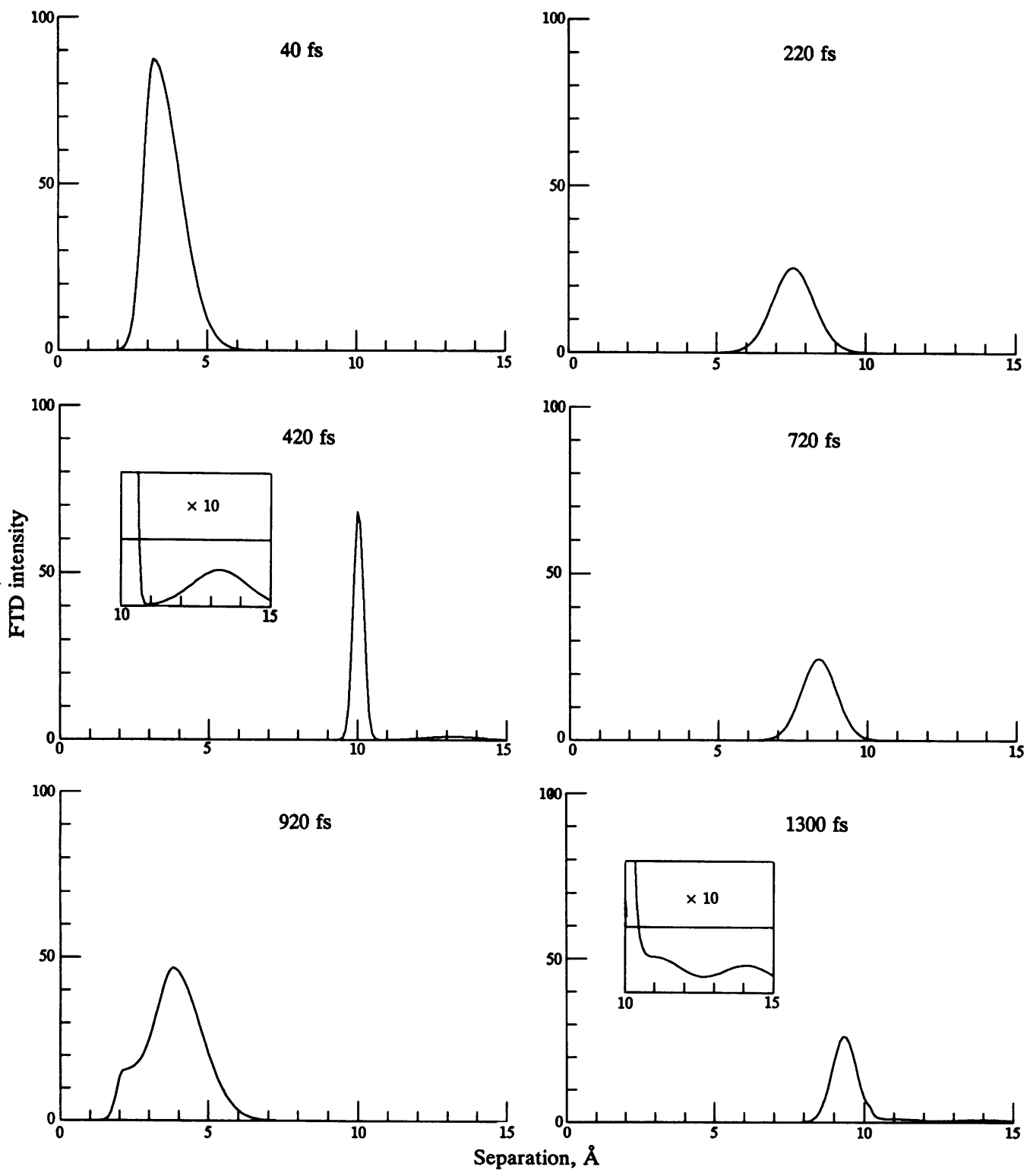

FIG. 4. Snapshots from the ERD curve of Fig. 3. The area under a peak is proportional to $n_{i j} Z_{i} Z_{j} / r_{i j}$ (see text); from $40 \mathrm{fs}$ to $420 \mathrm{fs}$, the area under the peak decreases in this $1 / r$ relationship. Peak width (full width at half-maximum) is proportional to velocity times the time span of the electron burst ( $50 \mathrm{fs}$ in this calculation). At $220 \mathrm{fs}$, excited $\mathrm{NaI}$ moves past the avoided crossing and $\approx 10 \%$ enters the ground state potential well. At $920 \mathrm{fs}$, ground state $\mathrm{NaI}$ is evident at $2 \AA$. At $1300 \mathrm{fs}$, dissociating ground state $\mathrm{NaI}$ appears at $14 \AA$ and excited NaI nears the turning point after another $\approx 10 \%$ has dissociated (peak at $11 \AA$ ). (Insets) A 10-fold magnification of a portion of the ERD. to ensure that on average an incident electron would experience a single elastic collision (16).

In the FTD experiment, visible/UV light and a photocathode with $10 \%$ quantum efficiency yield $10^{12}$ electrons that are to collide with the molecular beam. Both the molecular beam and the electron burst will have diameters of the order of 0.01 $\mathrm{cm}$; the electron beam will therefore encounter a volume of vapor of about $10^{-6} \mathrm{~cm}^{3}$. If we use the $\mathrm{NaI}$ experiment mentioned above as an example, we obtain a density of 2.8 $\times 10^{-7} \mathrm{~g} / \mathrm{cm}^{3}$ [vapor pressure of $100 \mathrm{mtorr}(1$ torr $=133 \mathrm{~Pa})$ at $600^{\circ} \mathrm{C}$. Taking the mass thickness parameter for $10-\mathrm{keV}$ electrons incident on $\mathrm{NaI}$ to be of the order of $10^{-3} \mathrm{mg} / \mathrm{cm}^{2}$ (29) leads us to conclude that multiple scattering constitutes no problem for FTD. Nearly $10^{10}$ electrons will be scattered, and this is two orders of magnitude greater than the number of electrons scattered in the picosecond film experiment. One has to take into account the optimization of the signal-tonoise ratio when considering the resolution element of solid angle (and the diffraction $s$ parameter) and the number of
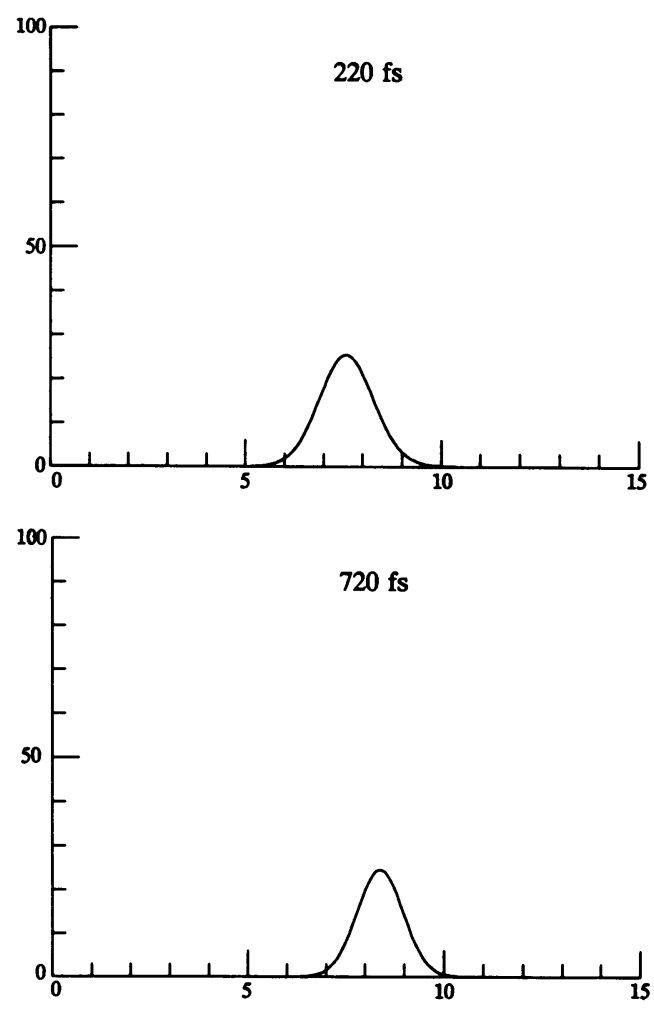
electrons that gives the best resolution. The number of pulses that will be used for averaging is determined by our laser repetition rate and sensitivity. In ordinary electron diffraction, the number of electrons is about $10^{13}$, typically for tens of seconds exposure. The sensitivity of the FTD experiment that we have described promises to be high enough for observing the femtosecond electron diffraction pattern of chemical reactions.

\section{Applications}

There are many applications for FTD. First, we consider the simple $\mathrm{NaI}$ reaction. $\mathrm{NaI}$ may be promoted to the covalent potential energy surface by using the femtosecond $t=0$ pulse (see Fig. 2). The oscillations of the activated complex within this covalent/ionic potential well, and its subsequent decay through the avoided crossing at $7 \AA$, have been observed with FTS $(7,9)$. With FTD, it will be possible to record the motion per se and obtain directly the different trajectories, as discussed below.

Because the atomic numbers of sodium and iodine are relatively large, $\mathrm{NaI}$ should be an excellent scatterer. The area underneath a maximum on the experimental radial distribution (ERD) curve is related to the atomic number $(Z)$ and the internuclear separation $\left(r_{i j}\right)$ by $n_{i j} Z_{i} Z_{j} / r_{i j} ; n_{i j}$ is the number of times the distance $r_{i j}$ occurs, and in this case $n_{i j}=$ 1. Because sodium is much smaller than iodine, it is possible that the ERD peak will show a doublet. This effect is referred to as intraatomic multiple scattering and is essentially due to differences in travel time (phase shift) between electrons passing by heavy nuclei versus light nuclei.

In the FTD experiment, the probe pulse will initiate an electron burst by striking the photocathode. By sequentially delaying the generation of the electron burst with respect to $t=0$, a series of diffraction snapshots will be taken. As shown in Fig. 3, over a time span of 0.5 ps, a peak moves from $2.8 \AA$ to $7 \AA$ in the simulated experimental radial distribution curve of FTD.

Over longer time spans, the FTS experiments show a signal decay as the activated complex "goes through" the avoided crossing (see Fig. 2). This behavior will be revealed in the ERD curve as a series of peaks moving out beyond $7 \AA$ (see Figs. 3 and 4). The peak area $\times r$ will decay sharply each time the separation goes past $R \approx 7 \AA$. As noted in Figs. 3 and 4 , the parts of the trajectories that go to the ground state can also be observed.

There are many other reactions to be studied and we believe that a wealth of information will be at hand for structural femtochemistry of elementary and complex reactions. It does not escape our attention that this technique will be quite general and applicable to a variety of reactions and conformational changes in different phases. There is one additional feature that is perhaps interesting. The initial pulse, if polarized, establishes an alignment, and this alignment will be maintained in the femtosecond time domain as the rotations of molecules on this time scale are negligible. [This feature has been explored in FTS $(30,31)$.] A dependence on the angle between incident electron beam and direction of polarization of the pump pulse will be a feature of FTD that can provide the angular distribution.
This is contribution no. 8396 from the California Institute of Technology. This work was supported by a grant from the National Science Foundation. We are grateful to Professor V. Schomaker for many helpful discussions.

1. Zewail, A. H. (1988) Science 242, 1645-1653.

2. Khundkar, L. R. \& Zewail, A. H. (1990) Annu. Rev. Phys. Chem. 41, 15-60.

3. Zewail, A. H. \& Bernstein, R. B. (1988) Chem. Eng. News 66, 24-43.

4. Baumert, T., Bühler, B., Thalweiser, R. \& Gerber, G. (1990) Phys. Rev. Lett. 64, 733-736.

5. Bowman, R. M., Dantus, M. \& Zewail, A. H. (1990) Chem. Phys. Lett. 174, 546-552.

6. Walkup, R. E., Misewich, J. A., Glownia, J. H. \& Sorokin, P. P. (1990) Phys. Rev. Lett. 65, 2366-2369.

7. Mokhtari, A., Cong, P., Herek, J. L. \& Zewail, A. H. (1990) Nature (London) 348, 225-227.

8. Zewail, A. H. (1991) Structure and Dynamics of Reactive Transition States: Faraday Discussion (Royal Soc. Chem. Letchworth, U.K.), Vol. 91.

9. Rose, T. S., Rosker, M. J. \& Zewail, A. H. (1989) J. Chem. Phys. 91, 7415-7436.

10. Shank, C. V. (1986) Science 233, 1276-1280.

11. Stearns, D. G., Wiedwald, J. D., Cook, B. M., Hanks, R. L. \& Landen, O. L. (1989) Rev. Sci. Instrum. 60, 363-367.

12. Murnane, M. M., Kapteyn, H. C., Rosen, M. D. \& Falcone, R. W. (1991) Science 251, 531-536.

13. Van Wonterghem, B. \& Rentzepis, P. M. (1990) Appl. Phys. Lett. 56, 1005-1007.

14. Frankel, R. \& Forsyth, J. (1989) Science 204, 622-624.

15. Harris, C. B., Ippen, E. P., Mourou, G. A. \& Zewail, A. H. (1990) Ultrafast Phenomena VII (Springer, New York).

16. Mourou, G. A. \& Williamson, S. (1982) Appl. Phys. Lett. 41, 44-45.

17. El-Sayed-Ali, H. E. \& Mourou, G. A. (1988) Appl. Phys. Lett. 52, 103-104.

18. El-Sayed-Ali, H. E. \& Herman, J. W. (1990) Rev. Sci. Instrum. 61, 1636-1647.

19. Prokhorov, A. M. \& Schelev, M. Ya. (1989) Laser Focus World 25, 85-92.

20. Bradley, D. J. (1977) in Ultrashort Light Pulses, ed. Shapiro, S. L. (Springer, Berlin), p. 17.

21. Sibbett, W., Niu, H. \& Baggs, M. R. (1982) Rev. Sci. Instrum. 53, 758-761.

22. Kinoshita, K., Ito, M. \& Suzuki, Y. (1987) Rev. Sci. Instrum. 58, 932-938.

23. Tebo, A. R. (1989) Laser Focus World 25, 42-43.

24. Niu, H., Degtyareva, V. P., Platonov, V. N., Prokhorov, A. M. \& Schelev, M. Ya. (1989) Proc. SPIE Int. Soc. Opt. Eng. 1032, 79-85.

25. Hargittai, I. \& Hargittai, M., eds. (1988) Stereochemical Applications of Gas-Phase Electron Diffraction (VCH, New York), p. 191.

26. Sainov, N. A. (1988) Sov. Tech. Phys. Lett. (Engl. Transl.) 14, 731-732.

27. Ewbank, J. D., Paul, D. W., Schäfer, L. \& Bakhtiar, R. (1989) Appl. Spec. 43, 415-419.

28. Schäfer, L. \& Ewbank, J. D. (1988) Acta Chem. Scand. Ser. A 42, 358-366.

29. Grivet, P. (1972) Electron Optics (Pergamon, New York), pp. 638-640.

30. Zewail, A. H. (1989) J. Chem. Soc. Faraday Trans. 2 85, 1221-1242.

31. Dantus, M., Bowman, R. M., Baskin, J. S. \& Zewail, A. H. (1989) Chem. Phys. Lett. 159, 406-412. 